\title{
Energy dependence of infrared emission from azulene C-H stretching vibrations
}

\author{
Jichun Shi, Diane Bernfeld, ${ }^{a)}$ and John R. Barkerb) \\ Department of Atmospheric, Oceanic and Space Sciences, Space Physics Research Laboratory, University \\ of Michigan, Ann Arbor, Michigan 48109-2143
}

(Received 4 January 1988; accepted 11 February 1988)

\begin{abstract}
New data are described on the energy dependence of infrared fluorescence (IRF) from the $\mathrm{C}-\mathrm{H}$ stretch modes $\left(\sim 3050 \mathrm{~cm}^{-1}\right)$ of vibrationally excited azulene $\left(\mathrm{C}_{10} \mathrm{H}_{8}\right)$. An optoacoustic method was used to measure the absorbed laser energy and determine the number of excited molecules, while time-resolved infrared emission was recorded. The experimental uncertainties of $\sim 5 \%$ are much smaller than in previous work and the agreement between the experiments and the theory for the IRF intensity is excellent for total vibrational energies from 14000 to $33000 \mathrm{~cm}^{-1}$.
\end{abstract}

\section{INTRODUCTION}

All vibrationally excited molecules are capable of emitting infrared fluorescence (IRF). For diatomic molecules, the theory is generally acknowledged to be mature and capable of predicting the IRF intensity as a function of vibrational energy. ${ }^{1}$ For larger molecules, the theory is also well developed $^{2}$ and experimental verification (within relatively large experimental uncertainties) has been reported in at least three different studies. ${ }^{3,4}$ For polyatomics, the theoretical relationship between IRF intensity and vibrational energy has been used to interpret laboratory studies of energy transfer, ${ }^{5}$ as well as infrared emission observed from astronomical objects. ${ }^{6}$ Both the energy transfer results and the astronomical interpretations rely fully on the IRF emission theory.

Recently, laboratory investigations of energy transfer involving vibrationally excited azulene were carried out with the IRF technique ${ }^{7}$ and with the ultraviolet (UVA) technique. ${ }^{8}$ Both methods use laser excitation of azulene, followed by rapid internal conversion, to produce ground electronic state azulene with known vibrational energy. In both methods, time-resolved signals (IRF intensity, or absorbance) are acquired and averaged for signal/noise improvement. Both methods require calibration curves to determine vibrational energy (as a function of time) from the observed signals. The calibration curve for the IRF experiments was derived from the theory mentioned above (and verified by experiment ${ }^{3,7}$ ), while that for the UVA experiments was obtained empirically. ${ }^{9}$ Ideally, the two experiments on the same molecule under similar conditions should give similar results.

The experimental results from the two methods showed good general agreement, except for the detailed shape of the derived vibrational energy decays, which led to different conclusions regarding the possible energy dependence of $\langle\Delta E\rangle$, the average energy transferred per collision. Because of the difference in $\langle\Delta E\rangle$ energy dependence, Hippler et al., who used the UVA technique, suggested that the IRF experiments were in error, probably due to inadequacy of the theoretical calibration curve.

\footnotetext{
a) Postdoctoral Research Associate.

b) Address correspondence to this author.
}

As discussed below, the IRF emission theory is based on several approximations that seem to be well justified, including the ergodic hypothesis. If the difference in the energy transfer results signaled a breakdown of the ergodic hypothesis, the implications would be far reaching, since this hypothesis underlies RRKM theory and other statistical dynamical theories. This possibility (and the desire to determine the correct energy dependence of $(\Delta E\rangle)$ ) motivated the new experimental test of the IRF emission theory reported in this paper. ${ }^{10}$ The results of this recalibration are used in the accompanying paper ${ }^{11}$ to analyze the IRF decay curves and determine the energy dependence of $\langle\Delta E\rangle$ with higher accuracy.

\section{EXPERIMENTAL}

The purpose of this experiment is to measure the IRF intensity produced by a known number of azulene molecules excited to various vibrational energies. The number of excited molecules produced initially in a laser pulse depends on the absorbed laser energy, which is proportional to the product of the absorber concentration, the laser pulse energy, and the absorption cross section (in the limit of small absorption) ${ }^{12,13}$ The first two quantities depend on experimental conditions that can vary from day to day, and the latter two quantities depend on the excitation wavelength. In the earlier IRF experiments with azulene, ${ }^{3,7}$ each of the three variables was measured independently; propagation of the measurement errors for the three separate measurements gave $\sim 30 \%$ uncertainties in the final calibration curve. In the present experiments, absorbed laser energy is monitored directly with a single optoacoustic measurement, leading to substantially reduced uncertainty.

Azulene $\left(\mathrm{C}_{10} \mathrm{H}_{8}\right)$ photophysics ${ }^{14}$ are particularly favorable for these experiments. The $S_{1} \leftarrow S_{0}$ and $S_{2} \leftarrow S_{0}$ band origins ${ }^{15}$ are near 14284 and $28757 \mathrm{~cm}^{-1}$, respectively, and the bands are easily pumped with tunable visible and ultraviolet lasers. Internal conversion to the ground state occurs in $\sim 1.9 \mathrm{ps}^{16}$ and $\sim 3 \mathrm{~ns}^{17}$ from the $S_{1}$ and $S_{2}$ states, respectively, and intersystem crossing is completely negligible in both states. The $S_{2}$ state fluorescence quantum yield is only $\sim 3 \%$, while that for the $S_{1}$ state is $<10^{-6}$, showing a decisive violation of Kasha's rule. ${ }^{18}$ Isomerization to napthalene 
is exothermic, but relatively slow, ${ }^{19}$ and therefore does not interfere with the IRF experiments. The photophysics of the higher $S_{n}$ states are not as well characterized, but they presumably exhibit similarly clean photophysics.

A schematic of the experimental apparatus is shown in Fig. 1. The IRF cell contains only pure azulene vapor, while the optoacoustic cell contains $100 \pm 4$ Torr of argon in addition to azulene. Two cells were needed because the low vapor pressure of azulene is not sufficient for optoacoustic measurements. The azulene vapor pressure in both cells was controlled with reservoirs cooled by recirculating fluid from the same temperature-regulated bath (Neslab model RTE-5B, $\pm 0.1{ }^{\circ} \mathrm{C}$ ). Azulene pressure in the IRF cell was measured ( $\pm 0.1 \mathrm{mTorr})$ with a capacitance manometer $0-1$ Torr (MKS Baratron model 227) and the argon pressure was measured with a mechanical guage (Matheson model 63). Both cells could be evacuated to $<10^{-5}$ Torr with a glass high vacuum line fitted with an oil diffusion pump and "greaseless" O-ring stopcocks and connectors. Because of difficulties previously experienced in making accurate measurements of azulene vapor pressure, ${ }^{7}$ the vapor pressure was held constant at $6.2 \pm 0.1 \mathrm{mT}$ Torr for most runs. Azulene was obtained from Aldrich and GC/MS analysis showed $>99 \%$ purity with no detectable napthalene impurity; the azulene crystals were thoroughly degassed before each experiment and used without further purification.

Two different IRF cells were used in these experiments. Cell A was $25 \mathrm{~mm}$ o.d. $\times 28 \mathrm{~cm}$, and cell B was $50 \mathrm{~mm}$ o.d. $\times 26 \mathrm{~cm}$. Both cells were fitted with Suprasil fused silica windows for the laser beam and $25 \mathrm{~mm}$ diameter sapphire fluorescence windows. The optoacoustic cell was constructed from a $250 \mathrm{~cm}^{3}$ flask and had $25 \mathrm{~mm}$ diameter Suprasil windows. The microphone with integral preamplifier (Lec- tret 1206 or Knowles BT-1759) was supported $\sim 4 \mathrm{~cm}$ from the wall and $\sim 1 \mathrm{~cm}$ from the edge of the laser beam.

The azulene $S_{1} \leftarrow S_{0}$ band was pumped using a pulsed tunable dye laser (Lumonics Hyperdye 300 ) pumped by a $\mathrm{XeCl}$ excimer laser (Lumonics Hyperex 400). A KDP crystal (Inrad, Autotracker II) was used to double the visible laser frequency and obtain tunable ultraviolet to pump the azulene $S_{2}$ and $S_{4}$ bands. According to the manufacturer's specifications, the dye laser bandwidth is $<0.1 \mathrm{~cm}^{-1}$, but this linewidth was not measured in our experiments. In the experiments using ultraviolet light, a UV-transmitting black glass filter, and $/$ or a $1800 \mathrm{~g} / \mathrm{mm}$ diffraction grating was used to eliminate the fundamental from the doubled output. The laser beam was steered using quartz $90^{\circ}$ prisms and it was expanded to $\sim 1 \mathrm{~cm}^{2}$ diameter and collimated with a $1 \times 3$ Gallilean telescope to reduce the power density. The beam diameter was held constant with irises, because the optoacoustic microphone sensitivity depends on the acoustic wave frequency, which is controlled by the beam diameter and the sound speed. ${ }^{20}$ In most experiments, the visible laser pulse energy at the IRF cell was $\leqslant 10 \mathrm{~mJ}$ pulse $^{-1}$ and the ultraviolet laser pulse energy was about an order of magnitude lower.

Because two cells were used, the laser beam intensity was lower at the microphone than at the fluorescence window, due to (1) gas phase absorption along the optical path and (2) window losses. The laser light attenuation was determined by simultaneously measuring initial intensity (reflected from a Suprasil flat) and transmitted intensity with two pyroelectric detectors (Molectron P3-01). To determine gas-phase absorption cross sections, measurements were carried out using azulene at various pressures and cells of different pathlengths. Attenuation by the cell windows was determined by chilling the azulene reservoir with liquid

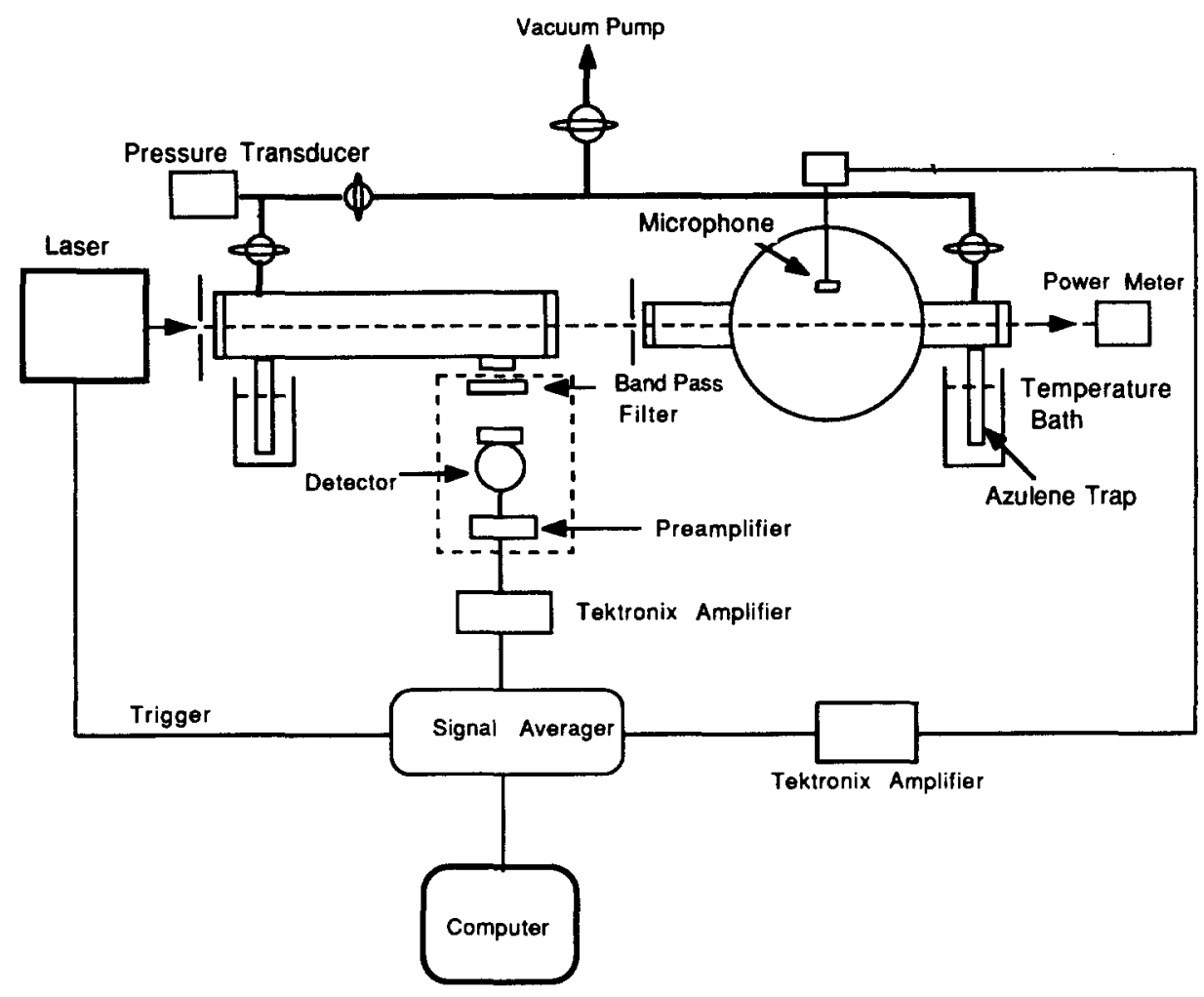

FIG. 1. Experimental apparatus. 

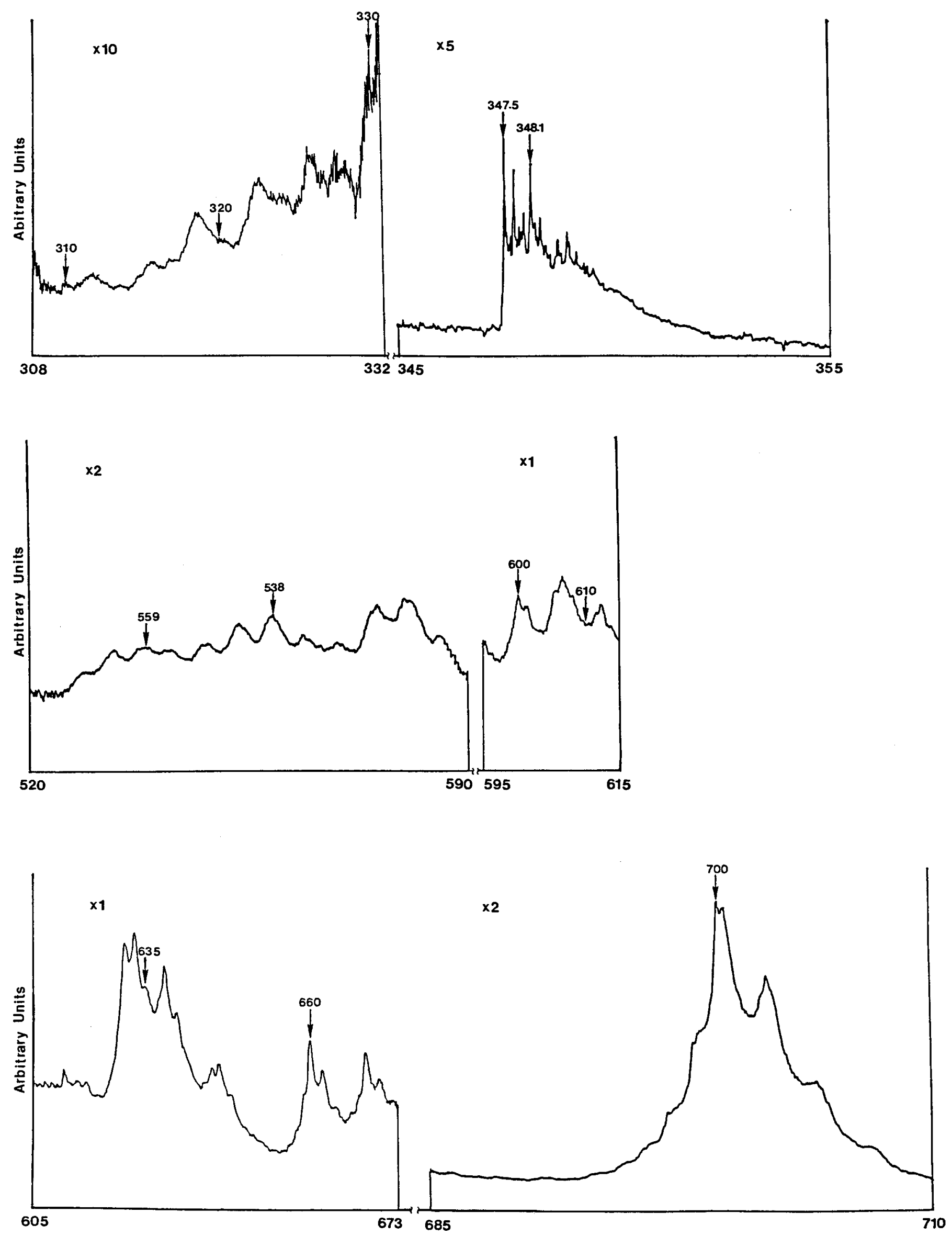

FIG. 2. Optoacoustic absorption spectrum of azulene. Experimental wavelengths (air) are indicated with arrows. 
nitrogen to remove the vapor, or by removing the cell windows and directly measuring the transmission.

The IRF emission was isolated with a bandpass infrared filter that transmits wavelengths in the range 2.5 to $3.8 \mu \mathrm{m}$. The IRF was observed with a $3 \mathrm{~mm}$ diameter $77 \mathrm{~K}$ InSb photovoltaic detector (Infrared Associates) and matched preamplifier. The output was amplified further (Tektronix AM-502 preamplifier) and captured with a two-channel digital oscilloscope (Le Croy model 9400). The observed time response of this system was tested with an LED emitter and was found to be $\sim 5 \mu$ s. The optoacoustic microphone output was amplified with a second preamplifier (Tektronix AM-502) and captured with the second channel of the digital oscilloscope.

In each experiment, both the IRF signal and the optoacoustic signal were simultaneously acquired and averaged using the digital oscilloscope. Although the optoacoustic signals could have been used directly with very little signal averaging, our procedure automatically accounted for possible changes in laser energy during the experiments, which usually lasted from 10 to $45 \mathrm{~min}$ at $25 \mathrm{~Hz}$ repetition frequency. At the conclusion of the experiments, the averaged IRF digital signal was transferred to a microcomputer and stored on floppy disks for later analysis; the optoacoustic signal intensity was also recorded.

Optoacoustic spectra were obtained with the optoacoustic cell filled with $100 \pm 4$ Torr of added argon. One channel of a boxcar integrator (Stanford Research Systems RS-250) was used to monitor the amplitude of the first maximum of the microphone signal as the laser was scanned. An absorbing calorimeter power meter (Scientech) was used to monitor the laser energy and its output was recorded with the second channel of the boxcar integrator. This signal was used to normalize the microphone signal and the resulting normalized spectra were recorded on a strip chart.

\section{RESULTS}

The optoacoustic spectra for azulene presented in Fig. 2 show the well-known lifetime broadening of the $S_{1}$ band and the relatively sharp lines present in the $S_{2}$ band. ${ }^{21}$ Also indicated in Fig. 2 are the 12 wavelengths investigated in the present study. Gas-phase absorption cross sections were measured at each of the wavelengths and the results are presented in Table $\mathrm{I}$. These results show good agreement with both solution-phase and gas-phase absorption spectra of azulene from other measurements. ${ }^{22}$ The large uncertainties at some wavelengths are due to the small absorptions that resulted from the small cross sections and the low pressures of azulene in the experiments; here, the uncertainties are not important in our experiments, because the gas-phase absorption is negligible.

The attenuation of the laser beam by two windows ( $>97 \%$ ) and the gas $(<3 \%)$ along the $14 \mathrm{~cm}$ optical path was included in calculating the relative number of excited molecules produced per laser pulse. Attenuation of the laser beam by the windows was found to be far more important than gas-phase absorption at all wavelengths, as shown in Fig. 3 and by inspection of the window transmission data in Table I. In the visible, the attenuation by two windows approximately equals the $\sim 16 \%$ expected from reflection at the four quartz/gas interfaces. In the ultraviolet, however, the attenuation is much larger, indicating that the windows have been affected by exposure to high laser powers in the presence of azulene. Under conditions of high azulene vapor pressure and high laser power (beam diameters of $\sim 1 \mathrm{~mm}$ ), opaque black burn spots permanently marred the windows.

The window transmission was combined with the small gas-phase absorption to produce the resulting laser beam transmission factor $F_{w}$ (see Table I) corresponding to the pressures used. Literature values for absorption cross sec-

TABLE I. Data summary.

\begin{tabular}{|c|c|c|c|c|c|c|c|}
\hline$\lambda(\mathrm{nm})^{\mathrm{a}}$ & $\langle E\rangle\left(\mathrm{cm}^{-1}\right)^{a}$ & $\sigma^{b}$ & $\sigma^{\mathbf{c}}$ & $F_{w}^{d}$ & $10^{-4} \tau^{-1}\left(s^{-1}\right)^{d}$ & $k^{e}$ & $I_{0}(\lambda)$ \\
\hline 700.0 & 14282 & $0.26(0.25)^{8}$ & 0.07 & 0.84 & $3.90(0.10)$ & $19.36(0.50)$ & $0.265(0.047)$ \\
\hline 660.0 & 15147 & $0.00(0.22)$ & 0.06 & 0.86 & $3.68(0.14)$ & $18.27(0.67)$ & $0.268(0.047)$ \\
\hline 635.0 & 15744 & $0.00(0.26)$ & 0.13 & 0.83 & $3.67(0.15)$ & $18.20(0.73)$ & $0.284(0.016)$ \\
\hline 610.0 & 16389 & $0.28(0.17)$ & 0.11 & 0.83 & $3.74(0.15)$ & $18.57(0.73)$ & $0.334(0.015)$ \\
\hline 600.0 & 16662 & $0.00(0.47)$ & 0.1 & 0.85 & $3.60(0.08)$ & $17.89(0.41)$ & $0.281(0.013)$ \\
\hline 558.8 & 17891 & $0.00(0.24)$ & 0.1 & 0.83 & $3.59(0.15)$ & $17.81(0.77)$ & $0.355(0.004)$ \\
\hline 538.0 & 18582 & $0.00(0.13)$ & 0.08 & 0.83 & $3.51(0.07)$ & $17.40(0.35)$ & $0.340(0.016)$ \\
\hline 348.1 & 28722 & $0.29(0.48)$ & 0.28 & 0.61 & $2.86(0.03)$ & $14.18(0.14)$ & $0.968(0.023)$ \\
\hline 347.5 & 28767 & $0.27(0.33)$ & 0.2 & 0.61 & $2.67(0.14)$ & $13.25(0.67)$ & $1.171(0.131)$ \\
\hline 330.0 & 30294 & $0.98(0.26)$ & 0.96 & 0.62 & $2.62(0.10)$ & $13.01(0.48)$ & $1.078(0.054)$ \\
\hline 320.0 & 31241 & $0.98(0.46)$ & 0.84 & 0.57 & $2.76(0.02)$ & $13.72(0.11)$ & $1.117(0.033)$ \\
\hline 310.0 & 32249 & $0.49(0.21)$ & 0.5 & 0.53 & $2.67(0.09)$ & $13.24(0.43)$ & $1.069(0.033)$ \\
\hline 281.6 & 35501 & $2.37(0.75)$ & 3.8 & $0.44^{f}$ & $0.84(0.05)^{f}$ & $11.73(0.70)$ & $1.163(0.093)$ \\
\hline 272.0 & 36754 & $13.70(0.86)$ & 19.9 & $0.48^{f}$ & $0.59(0.02)^{f}$ & $8.25(0.24)$ & $0.852(0.054)$ \\
\hline
\end{tabular}

"Laser wavelength (air) and laser photon energy (vacuum).

bUnits: $10^{-17} \mathrm{~cm}^{2}$.

${ }^{c}$ Reference 22. Units: $10^{-17} \mathrm{~cm}^{2}$.

${ }^{d} P_{\mathrm{Az}}=6.2 \mathrm{~m}$ Torr, unless noted otherwise.

'Units: $10^{-11} \mathrm{~cm}^{3} \mathrm{~s}^{-1}$.

${ }^{\mathrm{r}} P_{\mathrm{Az}}=2.1 \mathrm{~m}$ Torr.

$\pm 1 \sigma$ uncertainties in parentheses. 


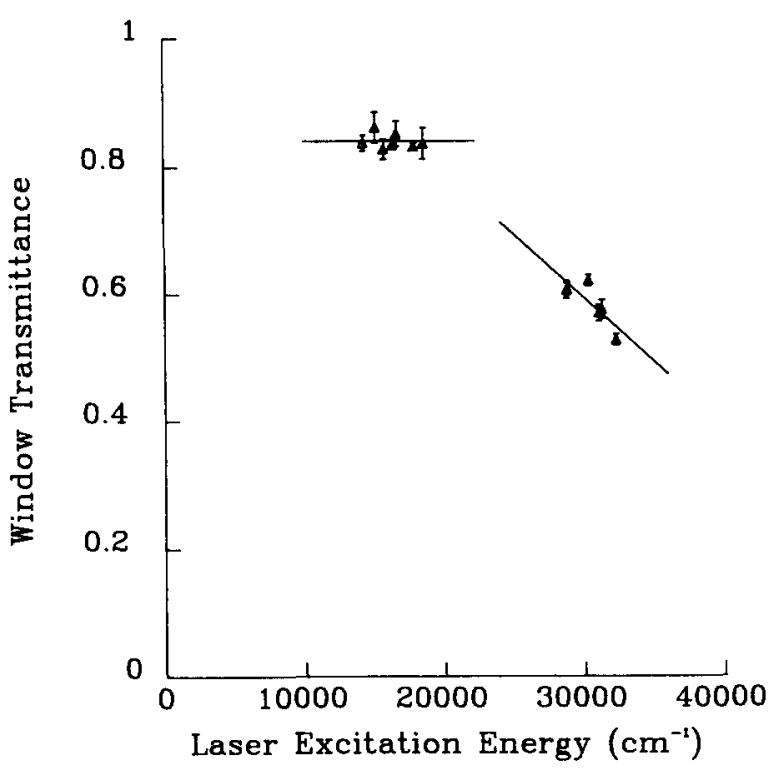

FIG. 3. Window transmission function.

tions were used for the $S_{1}$ band, but the values measured in this work were used for the ultraviolet gas-phase absorption.

The optoacoustic signal $S_{\mathrm{OA}}$ is proportional to the absorbed energy per unit length and inversely proportional to the heat capacity $C_{M}$ (which was held constant in the present experiments)

$$
S_{\mathrm{OA}}=-\frac{c^{\prime}}{C_{M}} \frac{d E_{A}}{d x}
$$

where $c^{\prime}$ is a proportionality constant. Use of the Beer-Lambert law for light absorption gives

$$
S_{\mathrm{OA}}=\frac{c^{\prime}}{C_{M}} \sigma N_{\mathrm{Az}} E_{1},
$$

where $\sigma$ is the azulene absorption cross section, $N_{\mathrm{Az}}$ is the azulene number density, and $E_{1}$ is the laser pulse energy. Equation (2) shows explicitly the relationship between $S_{\mathrm{OA}}$ and the three variables that were measured separately in the earlier investigations. ${ }^{3,7}$ The initial number density of excited molecules produced in front of the microphone is

$$
N^{*}=-\frac{1}{h v} \frac{d E_{A}}{d x}=\frac{C_{M}}{c^{\prime}} \frac{S_{\mathrm{OA}}}{h v}=c \lambda S_{\mathrm{OA}},
$$

where $v=1 / \lambda$ is the laser frequency and the other constants are absorbed in proportionality constant $c$. Tests were carried out to confirm that $S_{\mathrm{OA}}$ was directly proportional to $N_{\mathrm{Az}}$ and $E_{1}$ and there were no indications of multiple photon absorption. ${ }^{23}$ Note that the number density of excited molecules produced in front of the IRF window is $N^{*} / F_{w}$; this quantity is used to normalize the observed IRF signal.

A typical fluorescence decay signal observed in the present experiments is presented in Fig. 4. The baseline voltage prior to the laser pulse depends on detector bias voltage, amplifier offset, etc., in addition to the $300 \mathrm{~K}$ thermal emission from the interference filter, from the fluorescence cell walls, and a very small contribution from thermal azulene. After the pump laser fires, the IRF signal rises at detectorlimited time constant ( $\sim 5 \mu \mathrm{s})$ and then decays with a time

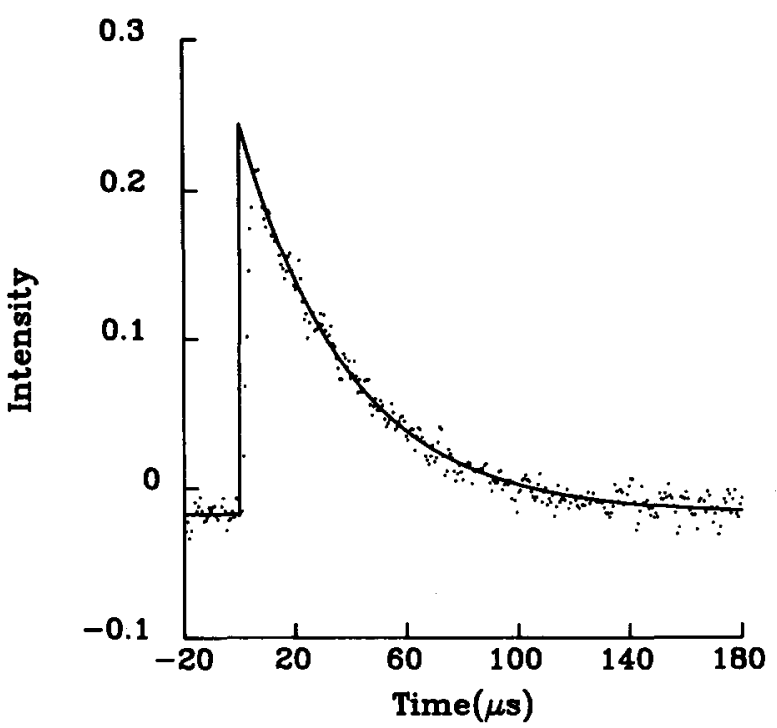

FIG. 4. Infrared fluorescence decay. $6.2 \mathrm{mT}$ Torr azulene excited at $320 \mathrm{~nm}$.

constant that depends on the excitation wavelength and the azulene pressure. As in earlier work, ${ }^{3,5,7}$ the decay was found to be essentially exponential after the initial $5 \mu$ s risetime and the solid line in Fig. 4 is a nonlinear least squares fit of the data assuming an exponential decay starting at $5 \mu \mathrm{s}$. The nonlinear least squares fit ${ }^{24}$ gives $\tau$, the time constant for the pseudoexponential decay, the intensity $I(t=5 \mu \mathrm{s})$, the zero offset of the signal, and the complete set of standard deviations and covariances.

Although the IRF decay is not exactly exponential, the deviations are very small and the exponential fit provides a good method for extrapolation from $I(t=5 \mu \mathrm{s})$ to $I(t=0) \equiv I_{0}$. To further test this extrapolation, IRF decays were measured for lower azulene pressures, where the extrapolation is not as long. All decay curves showed similar exponential behavior and the decay rate constants were found to be directly proportional to azulene pressure, as shown in Fig. 5. A phenomenological bimolecular decay rate constant can be defined as $k=\left(\tau N_{\mathrm{Az}}\right)^{-1}$. Values for $\tau$ and for $I(t=5$ $\mu \mathrm{s})$ were obtained by nonlinear least squares fits of each experimental run and extrapolated to obtain $I_{0}$; the uncertainty in $I_{0}$ was obtained through the use of the standard deviations and covariances determined in the least squares fitting. The weighted averages $k, \tau$, and $I_{0}$ for the runs at each laser wavelength are presented in Table I, along with the associated uncertainties.

It is worth noting that for azulene pressures less than $\sim 2 \mathrm{~m}$ Torr, the pressure measured with the capacitance manometer was systematically higher than literature data ${ }^{25}$ at the same temperature. The origin of this minor discrepancy was not identified, but several tests showed that the small pressure difference was not due to the presence of an unidentified impurity. Figure 5 shows a straight line passing through $\boldsymbol{P}=0$, indicating that if an impurity was present, its collision efficiency is the same as azulene. Measurements of $I_{0}$ and $S_{\mathrm{OA}}$ over a range of pressures showed direct proportionality to the meaured pressure, indicating that the IRF intensity and optoacoustic signal originate from the same 


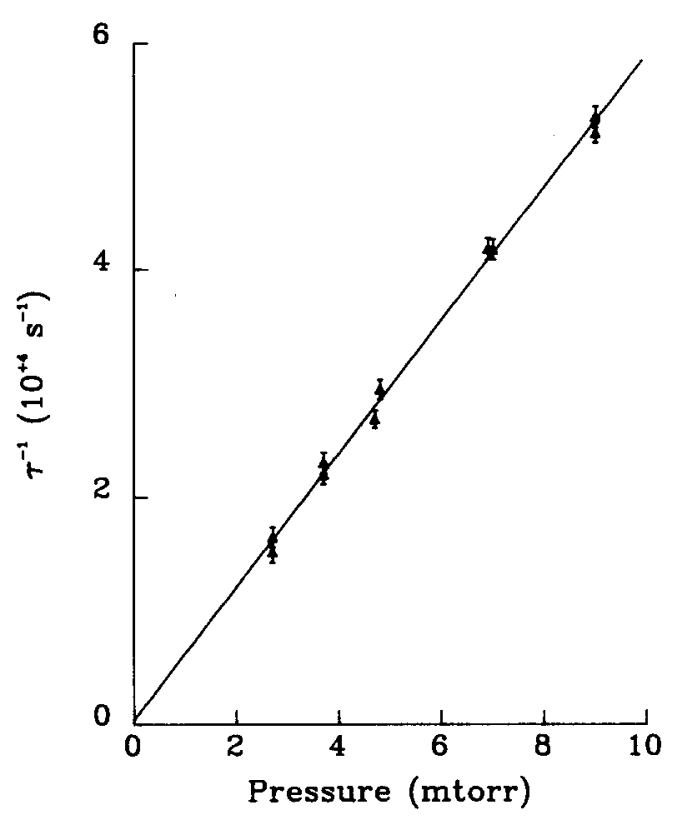

FIG. S. IRF decay inverse lifetime vs azulene pressure. $635 \mathrm{~nm}$ excitation. (Compare with Fig. 5 in Ref. 7.)

absorber (note that the most likely impurity is napthalene, and it does not absorb in the visible). These data taken together indicate that no impurity was present and the small discrepancy between our vapor pressure measurements and those in the literature has no effect on the present work.

The experimental calibration curve relating IRF intensity to laser excitation energy is obtained by normalizing $I_{0}$ according to the number of excited molecules produced in front of the IRF window

$$
I(\lambda)=\frac{I_{0}}{\left(N^{*} / F_{w}\right)}=\frac{1}{c} \frac{I_{0} F_{w}}{c \lambda S_{\mathrm{Oa}}},
$$

where the factors were defined above. The constant $c$ was chosen to make $I=1$ for $\lambda=337 \mathrm{~nm}$. The resulting values for $I(\lambda)$ are tabulated in Table $I$ and plotted in Fig. 6; the associated uncertainties $( \pm 1 \sigma)$ were obtained by a propagation of errors analysis using the uncertainties for the individual factors.

Included in Table $I$ (but not in Fig. 6) are $I(\lambda)$ data for wavelengths ( 272.0 and $281.6 \mathrm{~nm}$ ) corresponding to the azulene $S_{4}$ state which has unknown photophysical properties, but is likely to undergo rapid internal conversion. The $I(\lambda)$ data for this state indicate a sudden decrease of IRF intensity, relative to the $S_{2}$ state. If real, this decrease of intensity should be reflected in the IRF time decay data as an initial intensity increase, followed by the usual decay. However, the observed IRF showed only the usual decay. Thus we conclude that either internal conversion to $S_{0}$ is not the dominant photophysical process (producing less energy in vibration), or the large corrections needed for attenuation of the laser beam at these wavelengths has produced an experimental artifact. For this reason, we will discuss only the data corresponding to the $S_{1}$ and $S_{2}$ states, which have wellknown photophysical properties.

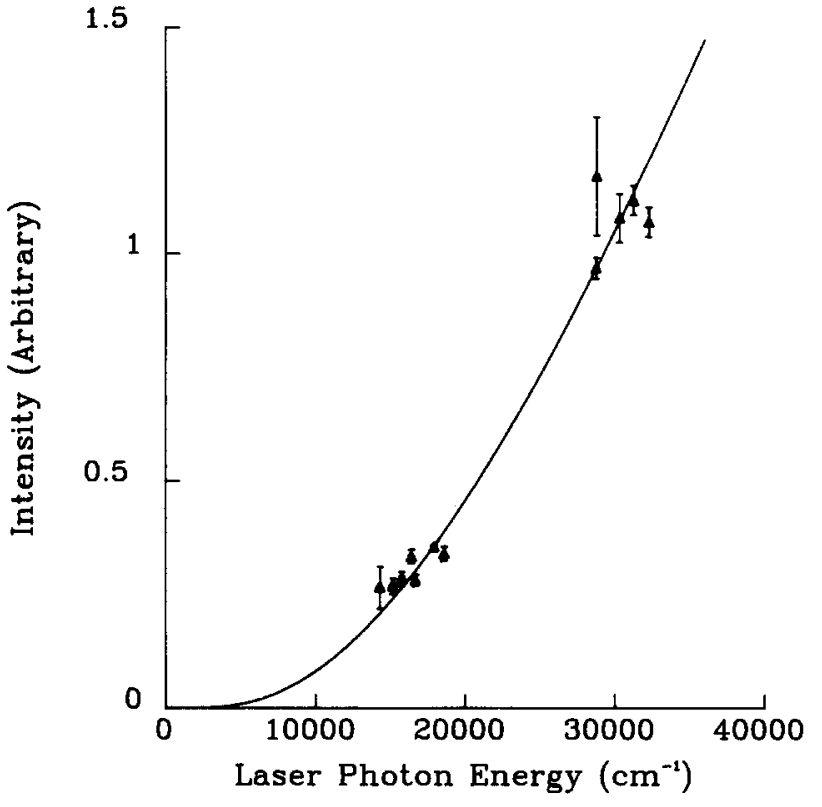

FIG. 6. IRF calibration curve. Experimental points and uncertainties $( \pm 1 \sigma)$ from Table I; solid line from Eq. (9), scaled to unity at $337 \mathrm{~nm}$ $\left(29674 \mathrm{~cm}^{-1}\right.$ ).

\section{DISCUSSION}

The theory of IRF emisson intensity for polyatomics ${ }^{2.3 .7}$ is based on the fundamental expression for a single oscillator $^{1}$

$$
I=h v_{v-1} A^{v, v-1} N_{v},
$$

where $v_{v-1}$ is the frequency and $A^{v, v-1}$ is the Einstein coefficient for $\Delta v=-1$ spontaneous emission from level $v$, and $N_{v}$ is the number density of molecules in level $v$. For moderate excitation energies, $A^{v, v-1}$ can be approximated by the following expression for anharmonic oscillators:

$$
A^{v, v-1} \simeq v\left(v_{v-1} / v_{0}\right)^{3} A^{1,0},
$$

where $v_{v-1}$ and $v_{0}$ are the frequencies of the $v-1 \leftarrow v$ and $0 \leftarrow 1$ transitions, respectively. For present purposes, the harmonic oscillator approximation is sufficient: $v_{v-1} \simeq v_{0}$ and $A^{v, v-1} \simeq v A^{1,0}$.

For polyatomic molecules, $N_{v}$ is calculated using the ergodic approximation, which assumes the energy is distributed statistically among all energetically accessible vibrational energy levels. Thus the average number of molecules with $v$ quanta in the emitting mode is

$$
N_{v}=N_{\text {ex }} \frac{\rho_{s-1}(E-v h v)}{\rho_{s}(E)},
$$

where $N_{\text {ex }}$ is the total number of density of excited molecules, $\rho_{s}(E)$ is the density of states for $s$ oscillators at vibrational energy $E$, and $\rho_{s-1}(E-v h v)$ is the density of states for the $s-1$ oscillators omitting the emitting mode and the vibrational energy contained in it. The total fluorescence observed through a bandpass filter is the sum of contributions from all energetically allowed levels up to $v_{\max }$ for all modes that emit in that wavelength range. The final expression for polyatomics is then written 
$I(E)=\frac{N_{\mathrm{ex}}}{\rho_{s}(E)} \sum_{i=1}^{\text {modes }} h v_{i} A_{i}^{1,0} \sum_{v_{i}=1}^{v_{\max }} v_{i} \rho_{s-1}\left(E-v_{i} h v_{i}\right)$

Here, $N_{\text {ex }}$ is the number of vibrationally excited molecules, $A_{i}^{1,0}$ is the Einstein coefficient for spontaneous emission for the $1 \rightarrow 0$ transition of the $i$ th mode, $h v_{i}$ is the energy of the emitted photon, and $\rho_{s}(E)$ and $\rho_{s-1}\left(E-v_{i} h v_{i}\right)$ are, respectively, the density of states for all $s$ oscillators at energy $E$ and that for the $s-1$ modes, omitting the emitting mode and the energy contained in it.

The harmonic oscillator approximation for Einstein coefficients is accurate within $\sim 10 \%$ for the first several levels of $\mathrm{C}-\mathrm{H}$ stretch modes. For several aromatic molecules, ${ }^{26}$ the anharmonic shift is about $115 \mathrm{~cm}^{-1}$ for each $\mathrm{C}-\mathrm{H}$ mode vibrational level, giving $\left(v_{1} / v_{0}\right)^{3} \simeq(2935 / 3050)^{3}=0.89$. The $v_{i}=1$ level contributes $>80 \%$ of the summation in Eq. (8) and the $11 \%$ error due to neglect of this anharmonicity is absorbed in the arbitrary scale factor used later to compare the experiments and theory, and therefore has no effect. The errors introduced by higher levels $\left(v_{i} \geqslant 2\right)$ amount to less than $3 \%$ and at energies $>35000 \mathrm{~cm}^{-1}$ and can be neglected.

The ergodic hypothesis has been tested for large molecules in unimolecular reaction studies, ${ }^{27}$ by laser pump/ probe experiments, ${ }^{28}$ and in infrared fluorescence experiments. ${ }^{29}$ The results indicate that intramolecular vibrational redistribution (IVR) is usually completed within picoseconds, even for $<3000 \mathrm{~cm}^{-1}$ of vibrational energy. In the present experiments, IRF can be observed only for vibrational energies greater than $3000 \mathrm{~cm}^{-1}$ (the $\mathrm{C}-\mathrm{H}$ quantum) and the time scale is microseconds, rather than picoseconds. Thus the ergodic hypothesis is expected to be valid.

In our experiments, a thermal distribution of molecules is excited by a laser photon of energy $h v_{1}$ and the IRF emission intensity is given by the averaged value of $I(E)$, as presented in Eq. (9),

$$
\left\langle I\left(h v_{1}, T\right)\right\rangle=\frac{1}{Q(T)} \int_{0}^{\infty} I\left(E+h v_{1}\right) \rho_{s}(E) \exp \left(-\frac{E}{k T}\right) d E,
$$

where $Q(T)$ is the vibrational partition function. In previous work, this expression was not calculated, but the average thermal energy $\left(979 \mathrm{~cm}^{-1}\right)$ was added to the photon energy to obtain (approximately) the total excitation energy.

In the present work, the densities of states were evaluated by exact count, using the Stein-Rabinovitch procedure ${ }^{30}$ with an energy grain of $25 \mathrm{~cm}^{-1}$. Harmonic oscillator vibrational frequencies were obtained from the vibrational assignment $^{31}$; anharmonicity and nonseparability probably have little importance for the 48 vibrational modes at the low energies considered here. ${ }^{32}$ The summations in Eq. (8) were evaluated explicitly and the integral in Eq. (9) was calculated numerically, for $T=300 \mathrm{~K}$. The calculated values for $\left\langle I\left(h v_{1}, T\right)\right\rangle$ were scaled to unity at $337 \mathrm{~nm}$ and are presented as the solid line in Fig. 6.

In previous work, the densities of states were evaluated using the Whitten-Rabinovitch approximation ${ }^{33}$ and $\left\langle I\left(h v_{1}, T\right)\right\rangle$ was not evaluated, but was approximated by $I\left(E+E_{t}\right)$, where $E_{t}$ is the average thermal energy content of the azulene at room temperature. These small differences between the previous calculations and the more accurate expressions produce small quantitative differences.

In Fig. 6, the experimental data show excellent agreement with the IRF calibration curve calculated according to Eq. (9). All data points are within two standard deviations of the calculated curve and most points are much closer.

Several experiments were performed on sharp spectroscopic features in the $S_{2}$ band to determine whether ergodicity holds as expected on the microsecond time scale of the experiments. Limited sets of experimental runs were performed on two sharp features (28 722 and $28767 \mathrm{~cm}^{-1}$ ) near the band origin, and the results show agreement within two standard deviations. Considering the small number of experimental runs, this agreement is satisfactory and it tends to confirm that the IRF intensity does not depend significantly on the details of the spectroscopic transition that is pumped. This result is not surprising, considering the long time scale of the present experiments compared with known nanosecond lifetimes of the $S_{2}$ state and the expected picosecond time scale for IVR in the $S_{0}$ state. Even so-called "isolated" $S_{2}$ levels $^{34,35}$ are likely to undergo IVR on a time scale as slow as microseconds.

The excellent agreement between experiment and theory for azulene gives us confidence in the theory as a tool for prediction and interpretation. Recently, considerable work has been done in this laboratory and elsewhere on the interpretation of infrared emission from interstellar dust clouds. ${ }^{6,36-38}$ The IR emission spectra show distinct band structure with frequencies characteristic of polycyclic aromatic hydrocarbon (PAH) species and it has been hypothesized that the PAHs absorb starlight, undergo internal conversion, and then emit IRF. We have used the theory to draw conclusions about the likely size of the PAH emitters $(\sim 20$ 30 carbon atoms) and it has been estimated that $5 \%-10 \%$ of the cosmic abundance of carbon may be in the form of these species. The present experimental data give support to the IRF emission theory, which is a key element in this interpretation.

The present agreement between theory and experiment also confirms the basic analysis carried out in the earlier energy transfer experiments with azulene. In the course of the present work, new high accuracy data on energy transfer were obtained and those data are described in the following paper. ${ }^{11}$ Briefly, the results strongly support the near-linear dependence of $\langle\Delta E\rangle$ on vibrational energy that was deduced earlier from IRF data ${ }^{3,5,7}$ and they conflict with published UVA data ${ }^{6}$ that show little dependence on vibrational energy. However, a recent report of new UVA data for azulene excited to the $S_{1}$ band shows better agreement. ${ }^{39}$

\section{ACKNOWLEDGMENTS}

The authors thank the Department of Energy (Office of Basic Energy Sciences) for funding. Thanks also go to Bruce Bartlett for the gift of a microphone and technical advice. Special appreciation goes to Dr. Ludwig Brouwer and Dr. Jean-Michel Zellweger, who worked with J. R. B. to show the feasibility of the IRF calibration experiments using the optoacoustic method (while at SRI International). 
'G. Herzberg, Infrared and Raman Spectra (Van Nostrand, Princeton, 1945).

${ }^{2}$ J. F. Durana and J. D. McDonald, J. Chem. Phys. 64, 2518 (1977).

${ }^{3}$ M. J. Rossi and J. R. Barker, Chem. Phys. Lett. 85, 21 (1982).

${ }^{4}$ J.-M. Zellweger, T. C. Brown, and J. R. Barker, J. Chem. Phys. 83, 6261 (1985).

${ }^{5}$ For a review, see J. R. Barker, J. Phys. Chem. 88, 11 (1984).

${ }^{6}$ L. J. Allamandola, A. G. G. M. Tielens, and J. R. Barker, Astrophys. J. 290, L25 (1985)

${ }^{7}$ M. J. Rossi, J. R. Pladziewicz, and J. R. Barker, J. Chem. Phys. 78, 6695 (1983).

${ }^{8}$ H. Hippler, L. Lindemann, and J. Troe, J. Chem. Phys. 83, 3906 (1985).

${ }^{9}$ L. Brouwer, H. Hippler, L. Lindemann, and J. Troe, J. Phys. Chem. 89, 4608 (1985).

${ }^{10} \mathrm{~A}$ preliminary report of these results appeared in J. R. Barker, Deactivation of Large Molecules in the Electronic Ground State, 10th International Conference on Molecular Energy Transfer, Emmetten, Switzerland, 2328 August, 1987.

${ }^{11} \mathrm{~J}$. Shi and J. R. Barker, J. Chem. Phys. 88, 6219 (1988).

${ }^{12}$ N. Presser, J. R. Barker, and R. J. Gordon, J. Chem. Phys. 78, 2163 (1983); K. M. Beck, A. Ringwelski, and R. J. Gordon, Chem. Phys. Lett. 121,529 (1985)

${ }^{13}$ J. R. Barker and B. M. Toselli, in Photothermal Investigations of Solids and Fluids, edited by Jeffrey A. Sell (Academic, New York, in press).

${ }^{14}$ D. G. Gillespie and E. C. Lim, J. Chem. Phys. 68, 4578 (1977).

${ }^{15} S_{1}$ origin: T. Suzuki and M. Ito, J. Phys. Chem. 91, 3537 (1987); $S_{2}$ origin: M. Fujii, T. Ebata, N. Mikami, and M. Ito, Chem. Phys. 77, 191 (1983).

${ }^{16}$ C. V. Shank, E. P. Ippen, O. T. Tesche, and R. L. Fork, Chem. Phys. Lett. 57, 433 (1978).

${ }^{17}$ A. E. W. Knight and B. Selinger, Chem. Phys. Lett. 12, 419 (1971); Y. Hirata and E. C. Lim, J. Chem. Phys. 69, 3292 (1978).

${ }^{18} \mathrm{~N}$. Turro, Modern Molecular Photochemistry (Benjamin/Cummings, Menlo Park, CA, 1978); S. Murata, T. Toda, and K. Kokuban, Chem. Phys. Lett. 13, 101 (1972); 15, 152 (1972).

${ }^{19}$ M. Comtet and H. O. Mette, Mol. Photochem. 2, 63 (1970); L. Brouwer and $\mathrm{J}$. Troe (to be published).
${ }^{20}$ J. Barker, L. Brouwer, R. Patrick, M. J. Rossi, P. L. Trevor, and D. M. Golden, Int. J. Chem. Kinet. 17, 991 (1985).

${ }^{21}$ G. R. Hunt and I. G. Ross, J. Mol. Spectrosc. 9, 50 (1962).

${ }^{22}$ UV Atlas of Onganic Compounds (Butterworths, London, 1966).

${ }^{23}$ For multiple photon absorption by azulene, see M. Damm, H. Hippler, and $J$. Troe (to be published).

${ }^{24}$ P. R. Bevington, Data Reduction and Error Analysis for the Physical Sciences (McGraw-Hill, New York, 1969), p. 237.

${ }^{25}$ A. Bauder and H. H. Gunthard, Helv. Chim. Acta 45, 1699 (1962).

${ }^{26}$ R. L. Swofford, M. E. Long, and A. C. Albrecht, J. Chem. Phys. 65, 179 (1976).

${ }^{27}$ I. Oref and B. S. Rabinovitch, Acc. Chem. Res. 12, 166 (1979).

${ }^{28}$ V. E. Bondybey, Annu. Rev. Phys. Chem. 35, 591 (1984).

${ }^{29}$ H. L. Kim, T. J. Kulp, and J. D. McDonald, J. Chem. Phys. 87, 4376 (1987).

${ }^{30}$ S. E. Stein and B. S. Rabinovitch, J. Chem. Phys. 58, 2438 (1973).

${ }^{31}$ R. S. Chao and R. K. Kahnna, Spectrochim. Acta Part A 33, 53 (1977).

${ }^{32}$ For the effect of nonseparability and anharmonicities on small molecules, see J. R. Barker, J. Phys. Chem. 91,3849 (1987).

${ }^{33}$ G. Z. Whitten and B. S. Rabinovitch, J. Chem. Phys. 41, 1883 (1964).

${ }^{34}$ A. E. W. Knight (private communication).

${ }^{35}$ D. R. Demmer, J. W. Hager, G. W. Leach, and S. C. Wallace, Chem. Phys. Lett. 136, 329 (1987).

${ }^{36}$ F. C. Gillett, W. J. Forrest, and K. M. Merrill, Astrophys. J. 183, 87 (1973); S. P. Willner, in Galactic and Extragalactic Infrared Astronomy, edited by M. F. Kessler and J. P. Phillips (Reidel, New York, 1984), p. 37; L. J. Allamandola, ibid., p. 5.

${ }^{37}$ A. Leger and J. L. Puget, Astron. Astrophys. 137, L5 (1984).

${ }^{38}$ For references, see L. J. Allamandola, A. G. G. M. Tielens, and J. R. Barker, in Physical Processes in Interstellar Clouds, edited by G. E. Morfill and M. Scholer (Reidel, New York, 1986), p. 305.

${ }^{39} \mathrm{~J}$. Troe, Collisional Energy Transfer in Chemical Reactions, 10th International Conference on Molecular Energy Transfer, Emmetten, Switzerland, 23-28 August, 1987; J. Troe and H. Hippler (private communication). 